\title{
Cognitive Skills of Future Philologists: Content, Structure and Promising Directions of the Development
}

\author{
Ivan Dmitrievich Yunyk
}

Interregional Academy of Personnel Management, Ukraine, 03039, Kiev, Frometovskaya street, 2

\section{Doi:10.5901/mjss.2015.v6n4s2p73}

\section{Abstract}

The author of the article specifies the content of the cognitive skills of future philologists as one of the means of active obtaining and using knowledge in the context of today's information space. A structural-functional model of the phenomenon is developed. In particular, in its structure, three components - the motivational and volitional, the operational and procedural and the content and reflective ones - are singled out, and their microstructure is developed. Promising directions of the development of cognitive skills of future philologists are identified, and they are based on ideas concerning psychological mechanisms of the functioning of motivation, attention, memory, thinking and the decision-making theory, which determine the cognitive activity of the individual.

Keywords: cognitive skills, information, future philologists, motivation, attention, memory, interference, thinking, cognitive dissonance.

\section{Introduction}

The modern civilization entered a brand new stage of its development - the information (post-industrial) one. Rapid increase in the volume of information requires the creation of innovative technologies of its perception, processing, storage and use. These civilizational changes provide for adequate reorientation of teaching science to the search for efficient forms and methods of training future philologists with due regard to the requirements of the multilingual communication world space (Bakhov, 2013).

In recent decades, scientists directed their efforts to studying processes of mastering information in terms of the threefold structure "knowledge-skills-habits", what is quite controversial. In particular, there are several methodological positions concerning studying the subject of research, where this process is characterized by:

- $\quad$ consecutive transition of knowledge to skills, and then to habits ("knowledge $\rightarrow$ skill $\rightarrow$ habit");

- relatively independent systematicy of skills and habits, which are formed and developed according to their own psychological laws;

- consideration of skills as the sum of the accumulated knowledge and the system of habits;

- demonstration of certain identity of the notions "skill and habit" with the construction of the algorithm "skill $\rightarrow$ habit $\rightarrow$ complex skill", and so on.

Regardless of the existence of different methodological positions concerning studying the process of mastering information, scientists agree that habits as automated actions are characterized by certain stereotype and are difficult to change, whereas skills are characterized by flexibility and deliberateness. At that, skills and habits are always interconnected with knowledge, because their strength depends on reliance on theoretical knowledge (Ryzhykov, 2015). That is why, in this process, it may be appropriate to consider not only skills and habits, the quantity and the quality of knowledge of future philologists, but also the set of means of their active getting and using in the context of today's information space, which usually requires variability and creative approach to problem solving. The basis of this set consists of cognitive skills of future philologists.

\section{Method}

The basis of the methodology consists of theoretical methods of research (of analysis and synthesis, induction and deduction, extrapolation and modeling initial assumptions of psychology and pedagogy) aimed at determining the content, the structure and promising directions of the development of the cognitive skills of future philologists. 


\subsection{Studying the content of the cognitive skills of future philologists}

The term "cognitive skills", coined by scientist I. Zabrodina, is quite uncommon in psychological and pedagogical literature, although a number of authors argue that such skill as the "ability to learn" is one of the leading individual skills. Scientists agree that the inability to learn leads to learning results that are lower than those that could be achieved based on real possibilities, or to attaining success at the cost of extremely hard work (Bakhov, 2014; Petrunova, 2009).

With regard to studying the definition of the concept "cognitive skills", their closeness to cognitional and gnostical skills is quite noticeable. It was N. Sholokhova who outlined the difference between these concepts most tellingly (Sholokhova, 2006). Agreeing with their partly synonymous properties, she notes that cognitional skills cover not only the components of the direct learning process, but also ensuring this process. Instead, the term "gnostical skills" characterizes the individual cognitive aspect of the learning process. According to the determination of $\mathrm{E}$. Ganayeva (Ganayeva, 1999), cognitive skills represent the ability of an individual to acquire knowledge on his/her own. With cognitive skills, she includes the ability to:

- $\quad$ work with visualization and literary text;

- make observations and draw conclusions;

- model and form hypotheses on one's own;

- $\quad$ apply knowledge in practice;

- set up an experiment and, on its basis, to gain knowledge on one's own;

- explain phenomena and facts on the basis of existing theoretical knowledge;

- foresee the consequences of following theoretical instructions.

N. Sholokhova adheres to a similar position (Sholokhova, 2006). In her view, cognitive skills are universal in nature and are directly related to the acquisition by an individual of knowledge as a result of cognitive activity. However, she also proposes an alternative approach to considering cognitive skills that can be realized through determining the content of objects involved in cognitive activity. She identifies three separate "blocks" - the information one, the subject one and the block of interaction. The first block includes the content, the form, the medium and the type of information, the second cognitive abilities (perceptual, mnemic, intellectual heuristic and intellectual logical ones), and the third block is characterized from several positions, including:

- cognitive processes that provide a mechanism for turning information into knowledge (attention, imagination, thought, memory, etc.);

- information handling actions (perception, understanding, processing, encoding, memorization and reproduction);

- ensuring all types of activities carried out by the personality with information (conditions, means, ways, methods, etc.).

D. Bernstein, L. Penner, A. Clarke-Stewart and E. Roy (Bernstein et al., 2012) believe that cognitive skills are focused on the ability to perform higher mental processes such as reasoning, comprehension, problem solving and decision making. Instead, L. Bidenko (Bidenko, 2012), based on the etymology of the term "cognition" (learning theory), treats the content of cognitive skills in terms of the general learning process, i. e., the perception of information, its comprehension and assimilation, drawing inferences, making decisions, etc. Taking this into account, it is fairly expedient to consider cognitive complexity (multiplicity) as a feature of the cognitive sphere, which is embodied in the trend to represent phenomena due to the aggregate of independent constructs.

A number of scholars treats "cognitive complexity" as a psychological characteristic of the cognitive sphere of an individual, which reflects the degree of categorical differentiation of his/her consciousness, which, in turn, determines the specifics of classification and categorization of different life events. There is also an alternative definition of the phenomenon in question associated with the antagonism of the concepts of "cognitive complexity" and "cognitive simplicity". Accordingly, cognitive complexity involves the use of a large number of constructs (units of the system of worldview of an individual), and cognitive simplicity - the use of a small number of constructs that have limited connections between them (Vinokur, 1987; Petrunova, 2009).

It should be noted that cognitive complexity has two interrelated aspects: conceptual complexity and integrative complexity. The former is the degree of differentiation of constructs, and the latter - the ability to correlate different constructs as well as to create combinations, to synthesize and to integrate them. In psychological and pedagogical science, the thought about the interrelation of the cognitive complexity of future philologists with production of new, unusual ideas can be traced. In particular, the relative stability of the cognitive complexity of an individual is stressed, because the system of cognitive structures (i. e., knowledge storage systems and means of cognition) constantly evolves (Petrunova, 2009). 


\subsection{Studying the structure of the cognitive skills of future philologists}

Analysis of psychological and pedagogical literature showed that the structure of cognitive skills has not become an object of special scientific research yet. However, the need to clarify the structural components of the studied phenomenon is explained by the need for finding effective directions of its development.

\subsubsection{The first component of the cognitive skills of future philologists}

According to H. Eichenbaum (Eichenbaum, 2008), S. Zanyuk (Zanyuk, 2002), E. Ilyin (llyin, 2004), S. Danylyuk (Daniliuk, 2013) and other researchers, one of the most important factors of influence on any activity is motivation, i. e., the set of impelling factors, to which end, its activity to action is determined. The structure of motivation must include motives - the impelling cause of actions and deeds of the individual. Their main role is to create momentum and orientation to the aim. At that, motives are always in close interrelation with the aim (the internal impelling motive). On this occasion, M. Ginsberg and R. Wlodkowski (Ginsberg and Wlodkowski, 2009) indicate that a clear sense of the aim plays crucial role in carrying out any activities, including studying foreign languages. It helps to avoid unrealistic expectations and to efficiently plan one's future activities, anticipating possible obstacles in advance and building a system of intermediate aims. Agreeing with this, S. Zanyuk (Zanyuk, 2002) and E. Ilyin (llyin, 2004) emphasize that realization of intermediate aims is a strong motivating factor.

It should be noted that motivation is not limited to the set of motives and the aim, as some influence is exerted by personal and situational factors. That is exactly why the intensity of actual motivation depends not only on the strength of the motive, but also from the effect of its situational determinants, including the complexity of the tasks.

The functioning of the cognitive skills of future philologists also depends on will, since the success of assimilation and reproduction of information depends on such qualities of linguists as dedication, perseverance, organization, initiative and so on. Instead, negative volitional qualities (impulsiveness, laziness, lack of organization, etc.) that are antagonistic to the above-mentioned ones, impede the attainment of the aim. Manifestation of will is represented by mental processes, among which there are perception, processing and reproduction of the necessary information as well as thinking and imagination, which require the mobilization of the volitional efforts of an individual (llyin, 2004; Stolyarenko, 2000).

One should understand volitional efforts as the state of internal mental stress necessary for overcoming the obstacles in the way of attaining the aim, while motivation - as not a mechanical connection of the aim with the motive, but a product of reflected processes initiated by needs, desires and aspirations that are impelling factors of the activity of subjects. Needs as the motivational source of volitional activity act as basal mental regulators of any activity. If in desires as in conscious experiences of an internal need the aim is only outlined and is reflected in a problematic way, in aspirations it (the aim) acquires a categorical nature. It is in aspirations that volitional efforts manifest themselves for the first time, and they depend on the motivation level (Zanyuk, 2002).

Volitional efforts are divided into spontaneous and arbitrary. The former are innate and acquired, what can be exemplified by defense and orientation responses. In contrast to the spontaneous ones, arbitrary volitional efforts are closely related to thinking and to the implementation of the aim; moreover, they contain conscious motives. However, it should be noted that spontaneous volitional efforts are connected with motives, too, but are not identical with them (llyin, 2004).

Summarizing all the information stated above, in the structure of the cognitive skills of future philologists, it is advisable to single out the motivational and volitional component which consists of three interconnected microcomponents, namely of:

- motives;

- $\quad$ aims and intermediate aims;

- volitional efforts.

\subsubsection{The second component of the cognitive skills of future philologists}

Through cognitive skills, memorization (perception, processing, storage) and application of information take place. This implies the need to consider the perception process in more detail. L. Stolyarenko (Stolyarenko, 2000) notes that, unlike sensations, the consequence of which is the appearance of basic feelings (of cold, heat, thirst, etc.), perception always generates images.

The essence of the process of perception is the reflection of objects and phenomena in the totality of their properties. With the defining characteristics of this process, one can include constancy, objectivity, integrity, 
structuredness and generality. In particular, L. Vekker (Vekker, 1974) emphasizes that the interaction of memory, thinking and many other mental processes of an individual allow perceiving any object or phenomenon as integral, independent of the conditions, in the totality of their stable connections and relations.

According to the research by L. Stolyarenko (Stolyarenko, 2000), the process of building an image is of a reflex nature and occurs in three stages:

- $\quad$ receiving and selecting perceptible sensations of different modalities;

- assembling an integral image out of the sensations received;

- $\quad$ applying to the resulting image various techniques of mental processing.

Thus, the perception scheme looks as follows: stimulus $\rightarrow$ system of data processing $\rightarrow$ reaction; stimulus- $2 \rightarrow$ system of data processing-2 $\rightarrow$ reaction-2, etc. The result of a certain number of the above schemes is an updated image. It should be noted that, in our opinion, the structure of the image has to include constructs by which psychological and pedagogical science means something inaccessible to direct observation, obtained through logical deduction based on the properties that are observed (Cordwell, 2000).

Agreeing with the above scheme, L. Vekker (Vekker, 1974) adds that one of the major operations in the system of data processing is the collation of the constructs of an image. During this "reconstruction of the image", a number of contradictions appears, for example, between levels of differentiation and integration of sensor data, between perceptual and mnemonic images. In the elimination of these contradictions, J. Mestre and B. Ross (Mestre and Ross, 2011) perceive the prerequisites of the appearance of the processes of differentiation, integration and so forth.

A similar position on the stages of information processing can be found in N. Sholokhova (Sholokhova, 2006). According to her, these include:

- perception, evaluation and interpretation of information;

- construction of the initial design of the newly perceived information;

- screening minor constructs of the information;

- designing a model of the essential components of the information;

- construction of the content and the structure of the current information;

- determination of interfering factors that affect the design of the information;

- improving the process of designing the content of information through creative transformations;

- combining the content of the new information with the content of the previously acquired information.

S. Sokoryeva (Sokoryeva, 1984) also believes that, after the reception of information, an individual singles out its characteristics, dividing them into major and minor ones, formulates generalizations based on substantial characteristics, and finds elements that bind the newly perceived information with one acquired earlier.

Extrapolating all the information stated above on the structure of the cognitive skills of future linguists, we deem it appropriate to single out in it the operational and procedural component that imparts "efficiency" to the phenomenon studied. With the content of this component, one can include five constituents that create a sequential algorithm of thinking operations, namely:

- perception of a generalized image;

- differentiation of the constructs of the image;

- collation of the constructs of the image;

- combining the constructs;

- $\quad$ synthesis of the constructs.

\subsubsection{The third component of the cognitive skills of future philologists}

The need for the search for the next component is preconditioned by the need for the final approval of the new generalized image or for its rejection for correction in case of non-compliance with the aim pursued. In this aspect, special attention should be paid to the phenomenon of reflection, which should be understood as an individual's ability to single out, to analyze and to correlate his/her actions with a specific situation.

A proof of the correctness of an in-depth consideration of reflection can be found in the writings of J. Mestre and B. Ross (Mestre and Ross, 2011). In their opinion, the need for making changes in activities appears as a result of the fact that they do not lead to attaining the aim. It is worth noting that, in the process of the professional training of future philologists, only the ability to reproduce knowledge is usually subject to inspection and evaluation, while the ability to get it goes under the radar. Thus, all the attention is directed to the result of activities and not to the process.

Among the researchers of reflection, H. Schedrovitsky and his scientific work (Schedrovitsky, 1957) is worth attention. It was he who, within the framework of the "activity theory", developed a theoretical justification of the model of 
reflection, which is considered, first, as the process and the structure of activity, and, secondly, as a mechanism of natural development of activity. In addition, he was the first to single out the stages of the process of reflection: studying the situation, identifying problems in the activity and ascertaining their reasons, criticizing the old standard and developing a new one. It should be noted that one of the prerequisites for realization of reflection is, according to the scholar, the reflexive going out, i. e., fixing the activity, what, in turn, will allow to objectively evaluate its effectiveness.

In turn, S. Daniliuk (Daniliuk, 2013) notes that reflection includes the analysis of the thinking or of the activities of future philologists, critical attitude toward them, what allows to make decisions on one's own. This implies the appropriateness of the use in terms of reflection of the assumptions of the theory of cognitive dissonance. Thus, according to L. Festinger (Festinger, 1989), it is the decision making process that allows to find a direct connection between motivation and activity, creating the effect of fixing. During this fixation, search for cognitive constructs with consonant relations and screening cognitive constructs with dissonant relations takes place. The condition for high performance of reception, storage and reproduction of information is constant striving of future philologists for reducing the strength of cognitive dissonance and for, at least, temporary achievement of consonance.

It should be noted that one of the causes of the appearance of cognitive dissonance between constructs of a new generalized image can be getting additional information (Chornei et al., 2005). In particular, this cause is important for future philologists with a high level of cognitive complexity, as they are able not only to single out more properties of phenomena and processes, but also to see a situation thoroughly, what results in the need for searching for more information. Instead, individuals with a low level of cognitive complexity perceive information in a more stereotyped way. Confirmation of this view can be found in the works of O. Vynokur (Vynokur, 1987), who states that individuals with an undifferentiated style of representation much faster switch from information to conclusions (i. e., they are less sensitive to lack of facts).

Summarizing all the above, we can trace the relevance of singling out in the structure of the cognitive skills of future philologists the content and reflective component, and in its microstructure - the three constituents, namely:

- fixation of a new generalized image;

- collation of the new generalized image with the perceived one;

- approval or rejection of the new generalized image.

\section{Results}

Due to using theoretical research methods, including analysis, synthesis, induction and deduction, it was found that the cognitive skills of future philologists were not an innate, but an acquired universal system of conscious actions directed at memorization (perception, processing, storage) and application of information. They represent the psychological basis for the formation of all types of general skills of future professionals, since they are the "tool", by means of which the transformation of information into knowledge takes place.

By means of methods of extrapolation and of modeling the assumptions of psychological and pedagogical science, a ternary structure of the cognitive skills of future philologists, which includes the motivational and volitional, the operational and procedural and the content and reflective components, was developed. The microstructure of the motivational and volitional component consists of three interrelated constituents: motives; the aim and intermediate aims; volitional efforts. The operational and procedural component comprises five constituents that create a sequential algorithm of thinking operations, namely: perception of the generalized image; differentiation of the constructs of the image; collation of the constructs of the image; combining the constructs; synthesis of the constructs. The structure of the content and reflective component of the cognitive skills of future philologists consists of three constituents: fixation of a new generalized image; collation of the new generalized image with the perceived one; approval or rejection of the new generalized image.

\section{Discussion}

\subsection{Promising directions of the development of the motivational and volitional component of the cognitive skills of future philologists}

According to S. Zanyuk (Zanyuk, 2002), C. Cefai (Cefai, 2008), A. Wigfield (Wigfield, 2009) and other researchers, one should distinguish between resultative and procedural motivation. Thus, if the causative factor is represented not by the outcome, but by the activity process itself, which makes the person enjoy the activity, it is indicative of the presence of procedural motivation. Thus, A. Wigfield (Wigfield, 2009) notes that the above types of motivation closely interact, 
although the reward, which relates to resultative motivation, may reduce the strength of internal (procedural) motivation.

Instead, resultative motivation is related to outlining the aim, as well as to accomplishing intermediate tasks on the way to attaining it. That is exactly why resultative motivational mindset plays a decisive role in the determination of motivation, especially in situations, where procedural motivation triggers negative emotions. In this case, aims and intentions that determine final and intermediate tasks of the activity and thereby mobilize the energy potential of the individual, become particularly important.

S. Zanyuk (Zanyuk, 2002) and E. Ilyin (llyin, 2004) pay special attention to the phenomenon of overmotivation (excessive strength of motivation). In their opinion, it causes an increase in the level of activity and stress. It is experimentally proved that, as a result of that, the quality of activity initially improves, however, after reaching the highest showings, it begins to gradually decline. At that, the level of motivation, with which a person demonstrates the highest performance of the activity, is optimal. However, it should be noted that this "optimum" is non-static: it will be different for positive and negative motivation, in different types of approval (verbal, physical, symbolic) and so forth. This "optimum" depends on the interaction of four key factors: on the personality of the individual; on the complexity of the tasks; on the subjective assessment of the likelihood of success; on the attractiveness of the consequences of success.

One of the ways to avoid "overmotivation" or to increase the level of motivation to the desired level is motivational strength correction that is carried out through absolute commitment of the individual to the process of activity. In this case, the decisive role is played by the correlation between the received information and the conceived image of the future result: too much disagreement between them has a destructive impact on the process of activity, while optimal disagreement, on the contrary, arouses interest and stimulates the search activity of the personality. In terms of procedural motivation, volitional components become particularly important, as the conceived image of the future result starts having motivating force and becomes the aim, towards which the actions of the individual are directed (llyin, 2004; Mestre and Ross, 2011; Zanyuk, 2002).

\subsection{Promising directions of the development of the operational and procedural component of the cognitive skills of future philologists}

The efficiency of the functioning of the second component of the cognitive skills of a personality is most strongly affected by attention, memory, thinking and other mental processes of the individual, what implies the need to examine them in more detail. Works of many researchers in the field of psychology and pedagogy (Chiapas, 1987; Roda, 2011; Tsotsos, 2011) are dedicated to the study of the nature of attention and of its properties. In particular, according to J. Tsotsos (Tsotsos, 2011), attention is to be understood as the sum of the mechanisms that help to set oneself up for controlling search processes characteristic of perception and cognition. It follows that attention does not have its own product and can only improve the outcome of the functioning of other mental processes.

The phenomenology of the concept of "attention" allows singling out a lot of its types. In particular, by the criterion of regulation they distinguish spontaneous attention, which is not associated with the volitional efforts of the individual, and arbitrary attention, which is consciously controlled and regulated. In addition, J. Tsotsos (Tsotsos, 2011) proposes to single out post-arbitrary attention that follows arbitrary one and differs from it in that it is associated with the taken aim of the activity, does not require volitional efforts and is not triggered by certain stimuli. Post-arbitrary attention appears when, in the process of purposeful activity, not only the result of this activity, but also the process of its carrying out becomes important for the individual. In our view, the emergence of post-arbitrary attention should be connected with the appearance of procedural (internal) motivation.

Of great importance are also properties of attention, which include stability, concentration, distribution, range, switching and fluctuations. Stability of attention is the duration of maintaining its intensity, concentration of attention is its focus on a specific type of activity, which is accompanied by ignoring extraneous stimuli. By distribution of attention one usually means its simultaneous deployment onto several objects or types of activity, which are all important for the individual at the same time. The range of attention is the number of objects that are perceived by the personality simultaneously. Switching of the attention is its rapid transition from one object/operation to another. Finally, fluctuations are regular simultaneous involuntary changes in the degree of the intensity of attention. At that, only stability and fluctuations as two opposite properties are not subject to the conscious regulation of the personality (Chiapas, 1987; Roda, 2011).

The number and the composition of objects, at which the attention of the individual can be directed in the process of activity, depend, primarily, on how many receptors are involved in the process of the perception of one or another information: the more their number, the greater the number of objects that can be in the field of attention. It is therefore important to determine by means of which sense organs a particular object should be controlled, because it can be 
controlled in different ways. If, for carrying out some actions, distribution of attention among multiple objects is required, the degree of its concentration will change. Therefore, when creating an optimal pattern of attention distribution, one should take into account the significance of each object for the individual at a given moment of time as well as its dynamics.

An important role in the process of the development of cognitive skills is played by memory, which determines the speed and the reliability of knowledge digestion. According to the results of the research by R. Atkinson (Atkinson, 1980), I. Hoffman (Hoffmann, 1986), Rainer H. Kluwe, Gerd Lüer, Frank Rösler (Kluwe et al., 2003) and other psychologists, the structure of memory consists of three components: sensory register, short-term memory and long-term memory.

Sensory register is a separate component of the memory of a personality, through which the perception of information from outside by visual, auditory or tactile receptors, its filtering and transferring to short-term memory take place. One of the features of sensory register is brevity of the maintenance of a clear image of newly perceived information that may be changed or even completely erased in this register through the perception of the next portion of information. At that, the selection of "valuable" information takes place due to managing the process of perception on the part of attention. Information that is less valuable for the individual goes under the radar and gets eliminated, while "valuable" one is transferred to short-term memory, and sometimes even the right to long-term one (Atkinson, 1980; Eichenbaum, 2008; Leser, 1979).

Due to the work of memory, correspondence between physical parameters of stimuli that affect in sensory register relevant receptors of the individual and the state of the central nervous system of the individual is established. Then the perceived stimuli get some meaning, and the information they carry is interpreted. It should be noted that the duration of the stay of information in sensory register (after the disappearance of the influence of the corresponding modality on a receptor) is $200-400 \mathrm{~ms}$ - during this period, random testing of this information or its rejection takes place (Hoffmann, 1986).

Short-term memory of an individual is a component of limited capacity, where a copy of the information created on outlet of sensory register and of long-term memory is formed. The main features of short-term memory include processing a reduced number of "new" images (only those that are "important" for the individual) by activating the corresponding traces in long-term memory as well as brevity of the retention of a clear image.

The main short-term memory management processes are:

- storage, search and activation of information in memory;

- repetition;

- encoding;

- $\quad$ figurative representation (Atkinson, 1980; Eichenbaum, 2008; Hoffmann, 1986; Leser, 1979).

The first process of management of short-term memory (storage, search and activation of information in memory) provides for collocation of received stimuli with semantic concepts, which are represented in the individual's long-term memory, their recognition and reproduction. At that, it is experimentally proved that, to maximize the efficiency of the realization of this process, the number of received stimuli should not exceed the number that can be successfully processed by the individual's short-term memory in terms of its range. Violation of this condition renders any correct repetition impossible (Atkinson, 1980).

Repetition is repeated reproduction of information (internal or external). Due to this, information stays in an individual's short memory for a longer time, what, together with favorable conditions for encoding, makes its fixation more effective. In addition, each new repetition delays the obliteration of a trace in short-term memory, renewing it. The number of stimuli that can reside in short-term memory during repetition depends on several conditions, including:

- the capacity of an individual's short-term memory;

- $\quad$ easiness of the recognition of stimuli;

- the ability of the individual to group stimuli;

- $\quad$ the speed of the processes of memorization.

It should be noted that most of the information is lost in the early stages of memorizing; therefore, according to F. Lyozer (Leser, 1979), it is necessary to repeat information immediately after its perception, and only then to increase intervals between repetitions.

E. Ganayeva (Ganayeva, 1999) also noted that repetition played an important role in the formation of skills. In her view, the formation of any skill is a complex process of analytical and synthetic activity of the cerebral cortex of the brain. In the course of this process, associations between a task, knowledge that is necessary for its accomplishment, and application of the knowledge in practice are created and imprinted. At that, repeated actions significantly reinforce the aforementioned associations.

The process of encoding can run both automatically and in controlled search mode. The first variant takes place in 
case of a strong connection of stimuli with corresponding represented semantic concepts, and the second one involves the use of randomly oriented attention of an individual (Hoffmann, 1986; Kluwe et al., 2003).

Figurative representation, by means of which semantic concepts are represented, requires additional time for their understanding. At that, the speed of the formation of figurative representations is determined by individual difference of the sense of easiness of the visualization of semantic information (Hoffman, 1986).

An individual's long-term memory is a component of large capacity, in which, in the form of semantic concepts, information is stored. These concepts can be represented to reproduce memorized information as well as to recognize and to encode newly perceived information (Eichenbaum, 2008; Roda, 2011).

According to the way of memorizing, P. Zinchenkо (Зінченко, 1961) singles out arbitrary and spontaneous memory. At that, each of the above types of memory is characterized by its own specifics of assimilation of information. Thus, arbitrary memory involves purposeful memorizing information through volitional efforts, and spontaneous one "automatic" memorizing information that takes place without assigning any mnemic tasks.

Particularly noteworthy are also the ideas of Zinchenko (Zinchenko, 1961) about dependence of the productivity of memorizing information on the extent of formedness of active mental operations. Modeling them on the process of the formation of cognitive skills, it should be noted that in the early stages of their formation the efficiency of spontaneous memorizing information remains low, when their formedness is sufficient, it increases dramatically, and when a certain degree of automation appears, it drops.

In terms of the functioning of memory we should also consider the phenomenon of interference, which is related to the complexity of structuring information. According to F. Lyozer (Leser, 1979), the main causes of the appearance of interference include a limited capacity of an individual's short-term memory and inability of an individual's memory to perform necessary operations concerning processing corresponding information. At that, there is an opinion that delimiting proactive interference (impact of new information on one that has already been assimilated) and retroactive interference (impact of previous activities on new ones) is expedient. In this regard, H. Eichenbaum (Eichenbaum, 2008) notes that, in case of the identity of information, retroactive interference turns into facilitation.

From the perspective of the operational and procedural component of the cognitive skills of an individual, an important role is played by thinking, which has a number of distinguishing features, namely depth, range, flexibility, speed, and ability to be critical. The depth of thinking is the easiness of identification of essential properties and relations between objects and phenomena, and the range of thinking is the breadth of coverage of the maximum number of objects with a clear awareness of the relations between them. By the flexibility of thinking, the plasticity of the stream of thought of an individual is meant. The essence of this feature can be explained as the opposite of inalterability. The speed of thinking is seen in psychological and pedagogical science as the pace of the stream of thought and is often associated with the temperament of an individual. Thus, phlegmatic and melancholic persons, in whom inhibition processes prevail over excitement ones, are often characterized by a low or moderate pace of thinking. Sanguine persons, whose processes of excitation and inhibition are in certain equilibrium, are characterized by a relatively high pace of thinking. Choleric persons, in whom excitation processes significantly outweigh inhibition ones, are able to demonstrate the highest pace of thinking. Finally, the ability of thinking to be critical is the ability to control the stream of thought, what helps it to evolve and to improve. In particular, P. Tittle (Tittle, 2011) emphasizes that critical thinking is a prerequisite for finding appropriate means to achieve the goal.

\subsection{Promising directions of the development of the content and reflective component of the cognitive skills of future philologists}

The basis of the above component is the theory of decision-making, because the fixation of a new generalized image is the result of finding constructs that are in consonant relations with one another as well as of rejecting constructs with dissonant interrelations. At that, the magnitude of cognitive dissonance between these constructs depends on the number of "conflicting" constructs. Persons with high tolerance to "conflict" of constructs are usually satisfied with the existing state of things, ignoring the search for alternatives to achieve consonance. Instead, persons with low tolerance to "conflict" of constructs direct their mental efforts at overcoming the dissonance. It should be noted that, in the first type of personality, lack of captiousness during the formation of a new generalized image can usually be seen, while in the second type, inhibition of mental processes due to excessive strength of dissonance can occur (Ginsberg and Wlodkowski, 2009; Vekker, 1974).

Removal of dissonance between constructs takes place due to conscious rejection of constructs with less positive effects and their subsequent fixation. It should be noted that, immediately during activity, the above decisions do not always lead to the appearance of complete consonance between the constructs of an image because conflicting relations 
between the positive properties of a rejected image and the negative properties of a selected image on the one hand, and their imagined models - on the other hand usually occur. This happens even in case of decided advantage of the positive properties of the constructs of a selected image over the negative properties of the constructs of a rejected one (Festinger, 1989).

During the fixation of the constructs of a selected image, the strength of cognitive dissonance is in direct relationship to the significance of their properties for the individual. In addition, the positive properties of a rejected image leave in the mind of the individual "imprints" that represent at the same time a reflection of the negative properties of the selected image. In this case, the strength of cognitive dissonance is directly proportional to the degree of attractiveness of the properties of the rejected image. After making the decision about the fixation of an image, the possibility of the appearance and the strength of cognitive dissonance depend on how much the properties of the selected and the rejected images coincide. If the degree of their similarity is high, then the strength of cognitive dissonance will be low, and vice versa. If, however, the properties of the selected and the rejected images coincide completely, then the emergence of cognitive dissonance is inevitable (llyin, 2004).

It should be emphasized that if, during many attempts, the aspiration of future philologists to reduce the strength of cognitive dissonance and, at least partially, to achieve consonance is not satisfied, they have the desire to substitute the rejected image for the selected one, what usually leads only to an increase in the strength of cognitive dissonance. In contrast, if the reason for the increase of the strength of dissonance between cognitive elements is getting additional information, then the effect of changing the decisions made will be positive.

Thus, the main means of reducing dissonance and/or achieving consonance between cognitive constructs is search for positive (including hidden) properties of constructs in a selected image and increasing their attractiveness as well as search for negative (including hidden) properties of constructs in a rejected image and decreasing their attractiveness. In addition, reduction of the strength of cognitive dissonance is caused by an increase in the number of cognitive constructs with features of the selected and the rejected images. L. Festinger (Festinger, 1989) notes that an effective example of the above can be finding or imaginary creating constructs of the selected image, the properties of which are similar to positive properties of constructs of the rejected image.

\section{Conclusions}

Based on the assumptions of psychological and pedagogical science, one can single out a number of promising directions of the development of the cognitive skills of future philologists, including:

- creating optimum motivational force;

- activating procedural motivation;

- $\quad$ taking into consideration the properties of attention during the assimilation of information;

- $\quad$ using an optimum pattern of attention distribution;

- influencing the work of sensory register, short-term and long-term memory;

- using arbitrary and spontaneous memory;

- avoiding proactive and retroactive interference;

- $\quad$ taking into consideration the role of the features of thinking during the assimilation of information;

- reducing dissonance and/or achieving consonance between cognitive elements.

Expediency of using all the ideas listed above in order to develop the cognitive skills of future philologists requires careful experimental verification in variable conditions of activity, and, as a consequence, further working out the methodology of purposeful development of their cognitive skills. It should be noted that the proposed article does not cover all aspects of the explored problem. In particular, cognitive complexity as a feature of the cognitive sphere of future philologists and its interrelation with cognitive skills require an in-depth study.

\section{References}

Atkinson, R., (1980). Human Memory and the Educative Process. Moscow: Progress

Bakhov, I. S. (2013). Government Multicultural Policy in Canada in the Period of 1970-2000-s. Middle-East Journal of Scientific Research, 15 (10), 1450-1454.

Bakhov, I. S. (2014). Dialogue of Cultures in Multicultural Education. World Applied Sciences Journal, 29 (1): 106-109.

Bernstein, D., Penner, L., Clarke-Stewart, A., \& Roy, E. (2012). Psychology (9th ed.). Wadsworth: Cengage Learning.

Bidenko, L. (2012) Formation of the Cognitive Skills of Future Teachers of Russian through Means of Information Technology. Abstract of candidate's dissertation. Kherson state pedagogical university. Kherson, Ukraine

Cefai, C. (2008). Promoting Resilience in the Classroom. A Guide to Developing Pupils' Emotional and Cognitive Skills. London: Jessica 
Kingsley.

Chiapas, V. (1987). Determination of Individual Differences of the Main Properties of Attention. (candidate's dissertation). Vilnius University, Vilnius, Lithuania.

Chornei, R., Hans Daduna, V.M. and Knopov, P. (2005). Controlled Markov fields with finite state space on graphs. Stochastic Models, $21(4), 847-874$.

Daniliuk, S. (2013). Theoretical and Methodological Basics of the Formation of the Professional Competence of Future Philologists through Means of Internet Technologies. (abstract of candidate's dissertation). Bohdan Khmelnytsky Cherkassy National university, Cherkassy, Ukraine.

Eichenbaum, H. (2008). Learning and memory. New York - London: W.W.Norton Company.

Festinger, L. (1989). Theory of Cognitive Dissonance. St.Petersburg, Yuventa.

Ganayeva, Ye. (1999). Formation of the Cognitive and Communicative Skills of Teenagers in Historical and Local Lore Activities. (abstract of candidate's dissertation). Orenburg State Pedagogical University, Orenburg, Russia.

Ginsberg, M., \& Wlodkowski, R. (2009). Diversity and Motivation. Culturally Responsive Teaching in College (2nd ed.). San Francisco: Jossey-Bass A Wiley Imprint.

Hoffmann, I. (1986). Active Memory. Experimental Studies and Theories of Human Memory. Moscow, Progress.

Ilyin, Ye. (2004). Motivation and Motives. St.Petersburg: Peter.

Kluwe, R., Lüer, G., \& Rösler, F. (2003). Principles of Learning and Memory. Basel: Birkhäuser Verlag.

Cordwell, M. Psychology from A to Z: Glossary, Year 2000. (2015, February 17). In National psychological encyclopedia. Retrieved February 17, 2015, from http://vocabulary.ru/dictionary/479/word/konstrukt

Leser, F. (1979). Memory Training. Moscow, Mir.

Mestre, J., \& Ross, B. (Eds.). (2011). The Psychology Of Learning And Motivation. Cognition in Education (Vol. 55). San Diego: Elsevier Academic Press.

Petrunova, T. (2009). Specifics of the Interrelation of the Emotional and the Cognitive Components of the Process of Mastering a Foreign Language in High School Students. (candidate's dissertation). Belgorod State University. Belgorod, Russia.

Ryzhykov, V. (2015). Economic Culture: Historical Aspect, Analysis, Prospects of the Development in the Context of the Formation of Economic Relations of Market Type. Actual problems of economics. 1 (163), P. 52-59.

Roda, C. (Ed.). (2011). Human Attention in Digital Environments. New York: Cambridge University Press.

Schedrovitsky, H. (1957). Language Thinking and its Analysis. Problems of Linguistics, 1, 449-465.

Sholokhova, N. (2006). Formation of the Cognitive Skills of Seventh- and Eighth-Graders in the Process of Learning Physics through Interactive Technologies. (candidate's dissertation). M. Dragomanov National Pedagogical University. Kiev, Ukraine.

Sokoryeva, S. (1984). Formation of Learning Skills and Habits in Students. Pedagogy of Higher School, 8, 65-71.

Stolyarenko, L. (2000). Basic Psychology. (3rd ed.). Rostov on Don: Fenix.

Tittle, P. (2011). Critical thinking : An appeal to reason. New York: Routledge.

Tsotsos, J. (2011). A Computational Perspective on Visual Attention. Cambridge MA: The MIT Press.

Vekker, L. (1974). Mental Processes. (Vol. 1). Leningrad. Leningrad University.

Wigfield, A. (2009). Handbook of Motivation at School (K. Wentzel, Ed.). New York: Routledge.

Vinokur, A. (1987). Influence of the Cognitive Complexity of the Social Perception of Senior Pupils on the Process of their Professional Self-Determination. (candidate's dissertation). Taras Shevchenko Kiev National University, Kiev, Ukraine.

Zanyuk, S. (2002). Psychology of Motivation. Kyiv, Lybid.

Zinchenko, P. (1961). Spontaneous Memorization. Moscow, APN RSFSR. 